Research Article

\title{
On Finite-Time Stability of Switched Systems with Hybrid Homogeneous Degrees
}

\author{
Bin Zhang (i) \\ School of Automation, Beijing University of Posts and Telecommunications, Beijing 100876, China \\ Correspondence should be addressed to Bin Zhang; zb362301@126.com
}

Received 8 June 2018; Accepted 30 July 2018; Published 8 August 2018

Academic Editor: Carlos-Renato Vázquez

Copyright ( 2018 Bin Zhang. This is an open access article distributed under the Creative Commons Attribution License, which permits unrestricted use, distribution, and reproduction in any medium, provided the original work is properly cited.

\begin{abstract}
The finite-time stability is investigated for switched nonlinear systems. It is assumed that each subsystem possesses a positive homogeneous Lyapunov-like function. The derivative of the function is with hybrid homogenous degrees. Three substantially different situations are considered and different sufficient conditions are provided, respectively. The utility of our result is illustrated through the study of a numerical example.
\end{abstract}

\section{Introduction}

Switched nonlinear systems are widely considered in engineering practice to represent a system with parameter jump and device conversion $[1,2]$. A switched system is essentially a hybrid system that consists of a family of subsystems and a switching law. The stability of the switching system is determined by both the individual stability of each mode and the logic of the switching law. The research achievements on the stability problem are fruitful $[3,4]$, especially on the switched systems; refer to the excellent works [5-7] and references therein.

Two general approaches to the stability problem of switched systems are common Lyapunov function (CLF) technique and multiple Lyapunov functions (MLFs) technique. The CLF technique has been effectively used in many situations $[8,9]$. A switched system with a CLF remains stable for any switching laws. Therefore, the CLF technique is naturally used when there is no a priori hypothesis of the switching law. However, the constructive problem of a CLF for general switched systems has not been solved.

MLFs technique relaxes the constraint conditions of CLF. In [10], it is shown that if the Lyapunov function of each mode is decreasing and the energy is decreasing at switching times, then the switched system is asymptotically stable. In [11], the MLFs condition is relaxed by introducing the concept of weak Lyapunov functions (WLFs). An extension of the invariance principle is provided relative to dwell time switched solutions.
In [12], union/intersection WLFs techniques are presented, where more accurate convergence region is obtained. In these works, maximal ratio coefficient is required among the Lyapunov functions. More specifically, for any subsystems $i$ and $j$, it is assumed that $V_{i} \leq \phi V_{j}$ with $\phi \geq 1$. However, it is not easy to get the estimation of $\phi$. Particularly, the existence of $\phi$ is not clear in many situations.

It is worth noting that homogeneous theory $[13,14]$ can give simplified conditions for stability analysis of switched nonlinear systems, where the value of $\phi$ is obtained accurately. In [15], stability problem of switched homogeneous systems is addressed using semitensor product of matrices and LMI conditions are achieved. In [16], homogeneous Lyapunov function is constructed and stability analysis via both CLF and MLFs is given. Some other results on this topic can be found in [17-19]. In comparison with the existing results where single homogeneous degree is considered, in this paper, we consider switched homogeneous systems with hybrid homogeneous degrees. That is to say, we consider homogeneous switched systems with Lyapunov function $\dot{V}_{i}(x) \leq-p V_{i}^{\theta}(x) \pm q V_{i}(x), 0<\theta<1$, rather than $\dot{V}_{i}(x) \leq$ $-p V_{i}^{\theta}(x)$. Recently, nonlinear systems with hybrid homogeneous degrees have attracted a considerable attention [20, 21]. However, such systems under switched conditions have not been investigated. This problem is considered in this paper. We extend the homogeneous results to the case with hybrid homogeneous degrees and sufficient conditions are obtained for finite-time stability. 


\section{Preliminaries}

Consider the following switched nonlinear system:

$$
\dot{x}=f_{\sigma}(x), \quad t \geq t_{0}, t_{0} \in \mathbb{R}
$$

where $x \in \mathbb{R}^{n}$ is the state vector and $\sigma(t):[0, \infty) \longrightarrow \mathscr{P}=$ $\{1,2, \ldots, p\}$ denotes the piecewise constant switching signal, which is continuous from the right; i.e., $\sigma(t+)=\lim _{s \downarrow t} \sigma(s)$. $\forall i \in \mathscr{P}, f_{i}$ is a smooth function with $f_{i}(0)=0$. Let $\left\{t_{1}, t_{2}, \ldots\right\}$ be the switching sequence. Then, it follows that $\sigma\left(t_{i}\right)$ is active in $\left[t_{i}, t_{i+1}\right)$. Throughout, we adopt the following assumption.

Assumption 1. For the switching sequence $\left\{t_{1}, t_{2}, \ldots\right\}$, there exists positive constant $\tau$ such that $t_{i}-t_{i-1}=\tau, \forall i \in\{1,2, \ldots\}$.

Definition 2. Let $x=\left(x_{1}, \ldots, x_{n}\right)^{T} \in \mathbb{R}^{n}$. A function $V$ : $\mathbb{R}^{n} \longrightarrow \mathbb{R}$ is called homogeneous of degree $\mu \in \mathbb{R}$ with respect to $r=\left(r_{1}, \ldots, r_{n}\right)^{T} \in \mathbb{R}_{+}^{n}$ if

$$
V\left(\lambda^{r_{1}} x_{1}, \ldots, \lambda^{r_{n}} x_{n}\right)=\lambda^{\mu} V\left(x_{1}, \ldots, x_{n}\right),
$$$$
\forall x \in \mathbb{R}^{n}, \forall \lambda \in \mathbb{R}_{+} .
$$

Definition 3. Let $x=\left(x_{1}, \ldots, x_{n}\right)^{T} \in \mathbb{R}^{n}$. A vector field $f(x)=\left(f_{1}(x), \ldots, f_{n}(x)\right)^{T}: \mathbb{R}^{n} \longrightarrow \mathbb{R}^{n}$ is called homogeneous with respect to $r=\left(r_{1}, \ldots, r_{n}\right)^{T} \in \mathbb{R}_{+}^{n}$ if for each $i \in$ $\{1, \ldots, n\}$

$$
\begin{aligned}
f_{i}\left(\lambda^{r_{1}} x_{1}, \ldots, \lambda^{r_{n}} x_{n}\right)=\lambda^{\kappa+r_{i}} f_{i}\left(x_{1}, \ldots, x_{n}\right), & \\
& \forall x \in \mathbb{R}^{n}, \forall \lambda \in \mathbb{R}_{+},
\end{aligned}
$$

holds for some constant $\kappa \geq-\min _{1 \leq i \leq n} r_{i}$. The constant $\kappa$ is called the degree of homogeneity. A time-invariant system $\dot{x}=f(x)$ is called homogeneous if its vector filed $f(x)$ is homogeneous.

Finite-time stability $[22,23]$ is considered in this paper and some definitions are provided as follows.

Definition 4. The origin is said to be a finite-time-stable equilibrium of (1) if there exists an open neighborhood $N \in$ $\mathbb{R}^{n}$ of the origin and a function $T: N \backslash\{0\} \longrightarrow(0, \infty)$, called the settling time, such that the following statements hold:

(1) Lyapunov stability.

(2) Finite-time convergence: for every $x_{0} \in N \backslash\{0\}$, the solution of (1) denoted by $\psi\left(x_{0}\right)$ with $x_{0}$ as initial condition is defined on $\left[0, T\left(x_{0}\right)\right)$, and $\lim _{t \rightarrow T\left(x_{0}\right)} \psi\left(x_{0}\right)=$ 0 .

The origin is said to be a globally finite-time-stable equilibrium if it is a finite-time stable equilibrium with $N=R^{n}$.

Assumption 5. For each $i \in \mathscr{P}$ of switched system (1), there exists $\mathscr{C}^{1}$ homogeneous function $V_{i}(x): \mathbb{R}^{n} \longrightarrow \mathbb{R}$ of degree $\mu \in \mathbb{R}$ with respect to $r=\left(r_{1}, \ldots, r_{n}\right)^{T} \in \mathbb{R}_{+}^{n}$ such that

$$
\begin{aligned}
\alpha \Lambda^{\mu}(x) & \leq V_{i}(x) \leq \beta \Lambda^{\mu}(x) \\
V_{i}(x) & \leq \phi V_{j}(x), \quad \forall i, j \in \mathscr{P}
\end{aligned}
$$

where $\Lambda(x)=\sum_{i=1}^{n}\left|x_{i}\right|^{1 / r_{i}}, \alpha=\min _{i \in \mathscr{P}} \min _{\Lambda(x)=1} V_{i}(x), \beta=$ $\max _{i \in \mathscr{P}} \max _{\Lambda(x)=1} V_{i}(x)$, and $\phi=\max _{i, j \in \mathscr{P}} \max _{\Lambda(x)=1}\left(V_{i}(x) /\right.$ $\left.V_{j}(x)\right)$.

Remark 6. Condition (4) is a direct corollary of function $V_{i}(x)$, in view of the homogeneous property. In (2), we choose $\lambda=1 / \Lambda(x)$; it follows that

$$
\begin{aligned}
V_{i}\left(x_{1}, \ldots, x_{n}\right) & =\frac{1}{\lambda^{\mu}} V_{i}\left(\lambda^{r_{1}} x_{1}, \ldots, \lambda^{r_{n}} x_{n}\right) \\
& =\Lambda^{\mu}(x) V_{i}\left(\xi_{1}, \ldots, \xi_{n}\right)
\end{aligned}
$$

where $\xi_{i}=\lambda^{r_{i}} x_{i}$. Let $\xi=\left(\xi_{1}, \ldots, \xi_{n}\right)^{T}$. We can get $\Lambda(\xi)=$ $\sum_{i=1}^{n}\left|\xi_{i}\right|^{1 / r_{i}}=\lambda \sum_{i=1}^{n}\left|x_{i}\right|^{1 / r_{i}}=\lambda \Lambda(x)=1$. Therefore, we conclude that $\alpha \leq V_{i}\left(\xi_{1}, \ldots, \xi_{n}\right) \leq \beta$, which implies (4) holds. Similarly, we have

$$
\frac{V_{i}(x)}{V_{j}(x)}=\frac{\Lambda^{\mu}(x) V_{i}\left(\xi_{1}, \ldots, \xi_{n}\right)}{\Lambda^{\mu}(x) V_{j}\left(\xi_{1}, \ldots, \xi_{n}\right)} \leq \max _{i, j \in \mathscr{P}} \max _{\Lambda(x)=1} \frac{V_{i}(x)}{V_{j}(x)}
$$

which implies (5) holds.

Remark 7. In the existing literature, switched systems with $\dot{V}_{i} \leq-p V_{i}^{\theta}$ for some $p, \theta \in \mathbb{R}$ are widely considered. Different kinds of sufficient conditions for switched stability have been presented. In this paper, we consider switched systems with hybrid homogeneous degrees, i.e., $\dot{V}_{i} \leq a V_{i}^{\theta}+b V_{i}$ for some $a, b, \theta \in \mathbb{R}$, and as far as we know, there are no results on this kind of switched systems. Specifically, the following two different situations are considered.

Assumption 8. For the homogeneous function $V_{i}(x), i \in \mathscr{P}$, defined in Assumption 5, we have

$$
\frac{\partial V_{i}}{\partial x} f_{i}(x) \leq-p V_{i}^{\theta}(x)+q V_{i}(x)
$$

where $p>0, q>0$, and $0<\theta<1$.

Assumption 9. For the homogeneous function $V_{i}(x), i \in \mathscr{P}$, defined in Assumption 5, we have

$$
\frac{\partial V_{i}}{\partial x} f_{i}(x) \leq p V_{i}^{\theta}(x)-q V_{i}(x)
$$

where $p>0, q>0$, and $0<\theta<1$.

Assumption 10. For the homogeneous function $V_{i}(x), i \in \mathscr{P}$, defined in Assumption 5, we have

$$
\frac{\partial V_{i}}{\partial x} f_{i}(x) \leq-p V_{i}^{\theta}(x)-q V_{i}(x)
$$

where $p>0, q>0$, and $0<\theta<1$.

\section{Main Results}

\subsection{Stability with (8)}

Lemma 11. Let $t^{\prime \prime}>t^{\prime} \geq t_{0}$. If function $y(t): \mathbb{R} \rightarrow \mathbb{R}$ satisfies

$$
\dot{y}(t)=-p y^{\theta}(t)+q y(t)
$$


for $p>0, q>0$, and $0<\theta<1$, then one gets that

$$
\begin{aligned}
y^{1-\theta}\left(t^{\prime \prime}\right)= & e^{(1-\theta) q\left(t^{\prime \prime}-t^{\prime}\right)} y^{1-\theta}\left(t^{\prime}\right) \\
& +\frac{p}{q}\left(1-e^{(1-\theta) q\left(t^{\prime \prime}-t^{\prime}\right)}\right) .
\end{aligned}
$$

Proof. Multiplying (11) by $e^{-q t}$, we get that

$$
e^{-q t} \dot{y}(y)-q e^{-q t} y(t)=-p e^{-q t} y^{\theta}(t)
$$

which implies

$$
\frac{d\left(e^{-q t} y(t)\right)}{d t}=-p e^{-q t} y^{\theta}(t)
$$

Let $z(t)=e^{-q t} y(t)$. Then, we have that

$$
\frac{d z}{d t}=-p e^{-(1-\theta) q t} z^{\theta}
$$

Integrating (15) on the time interval $\left(t^{\prime}, t^{\prime \prime}\right)$, we get that

$$
z^{1-\theta}\left(t^{\prime \prime}\right)=z^{1-\theta}\left(t^{\prime}\right)+\frac{p}{q}\left(e^{-(1-\theta) q t^{\prime \prime}}-e^{-(1-\theta) q t^{\prime}}\right)
$$

which implies

$$
\begin{aligned}
y^{1-\theta}\left(t^{\prime \prime}\right)= & e^{(1-\theta) q\left(t^{\prime \prime}-t^{\prime}\right)} y^{1-\theta}\left(t^{\prime}\right) \\
& +\frac{p}{q}\left(1-e^{(1-\theta) q\left(t^{\prime \prime}-t^{\prime}\right)}\right) .
\end{aligned}
$$

Theorem 12. Consider switched system (1). Assume that Assumptions 1-8 hold. Let $\Upsilon=(p / q)\left(\phi\left(e^{(1-\theta) q \tau}-1\right) /\right.$ $\left.\left(\phi e^{(1-\theta) q \tau}-1\right)\right)$ and $\Psi=e^{(1-\theta) q \tau} V_{\sigma\left(t_{0}\right)}^{1-\theta}\left(x\left(t_{0}\right)\right)-\Upsilon$. Then, the origin of (1) is locally finite-time stable for initial value $x\left(t_{0}\right)$ satisfying

$$
V_{\sigma\left(t_{0}\right)}\left(x\left(t_{0}\right)\right) \leq \frac{1}{e^{q \tau}}\left(\frac{p}{q} \frac{\phi\left(e^{(1-\theta) q \tau}-1\right)}{\phi e^{(1-\theta) q \tau}-1}\right)^{1 /(1-\theta)} .
$$

Moreover, the setting time is $T=t_{\Pi}$, where $\Pi=[\ln (-\Upsilon / \Psi) /$ $\left.\ln \left(\phi e^{(1-\theta) q \tau}\right)\right]$.

Proof. Using the homogeneous function $V_{i}(x), i \in \mathscr{P}$, we construct the multiple Lyapunov function $V_{\sigma(t)}(x)$ corresponding to switching law $\sigma(t)$. We can see that $V_{\sigma(t)}(x)$ is piecewise smooth.

For any $t \geq t_{0}$, there exists time interval $\left[t_{j-1}, t_{j}\right), j \geq 1$, such that $t_{j-1} \leq t<t_{j}$. Integrating differential inequalities (8) successively on the intervals $\left[t_{0}, t_{1}\right),\left[t_{1}, t_{2}\right), \ldots,\left[t_{j-1}, t\right]$, we can get

$$
\begin{aligned}
& V_{\sigma\left(t_{j-1}\right)}^{1-\theta}(x(t)) \leq e^{(1-\theta) q\left(t-t_{j-1}\right)} V_{\sigma\left(t_{j-1}\right)}^{1-\theta}\left(x\left(t_{j-1}\right)\right) \\
& \quad+\frac{p}{q}\left(1-e^{(1-\theta) q\left(t-t_{j-1}\right)}\right) \\
& V_{\sigma\left(t_{j-2}\right)}^{1-\theta}\left(x\left(t_{j-1}\right)\right) \leq e^{(1-\theta) q\left(t_{j-1}-t_{j-2}\right)} V_{\sigma\left(t_{j-2}\right)}^{1-\theta}\left(x\left(t_{j-2}\right)\right) \\
& \quad+\frac{p}{q}\left(1-e^{(1-\theta) q\left(t_{j-1}-t_{j-2}\right)}\right) \\
& \ldots
\end{aligned}
$$

$$
\begin{aligned}
& V_{\sigma\left(t_{0}\right)}^{1-\theta}\left(x\left(t_{1}\right)\right) \leq e^{(1-\theta) q\left(t_{1}-t_{0}\right)} V_{\sigma\left(t_{0}\right)}^{1-\theta}\left(x\left(t_{0}\right)\right) \\
& +\frac{p}{q}\left(1-e^{(1-\theta) q\left(t_{1}-t_{0}\right)}\right) .
\end{aligned}
$$

Taking into account condition (5), we obtain

$$
V_{\sigma\left(t_{i}\right)}^{1-\alpha}\left(x\left(t_{i}\right)\right) \leq \phi V_{\sigma\left(t_{i-1}\right)}^{1-\alpha}\left(x\left(t_{i}\right)\right)
$$

for any $i=1, \ldots, j-1$. Substituting (20) into (19) yields

$$
\begin{aligned}
& V_{\sigma\left(t_{j-1}\right)}^{1-\theta}(x(t)) \leq \phi e^{(1-\theta) q\left(t-t_{j-1}\right)} V_{\sigma\left(t_{j-2}\right)}^{1-\theta}\left(x\left(t_{j-1}\right)\right) \\
& \quad+\frac{p}{q}\left(1-e^{(1-\theta) q\left(t-t_{j-1}\right)}\right) \\
& \quad V_{\sigma\left(t_{j-2}\right)}^{1-\theta}\left(x\left(t_{j-1}\right)\right) \leq \phi e^{(1-\theta) q\left(t_{j-1}-t_{j-2}\right)} V_{\sigma\left(t_{j-3}\right)}^{1-\theta}\left(x\left(t_{j-2}\right)\right) \\
& \quad+\frac{p}{q}\left(1-e^{(1-\theta) q\left(t_{j-1}-t_{j-2}\right)}\right) \\
& \quad . \quad \\
& V_{\sigma\left(t_{0}\right)}^{1-\theta}\left(x\left(t_{1}\right)\right) \leq e^{(1-\theta) q\left(t_{1}-t_{0}\right)} V_{\sigma\left(t_{0}\right)}^{1-\theta}\left(x\left(t_{0}\right)\right) \\
& \quad+\frac{p}{q}\left(1-e^{(1-\theta) q\left(t_{1}-t_{0}\right)}\right) .
\end{aligned}
$$

It follows that

$$
\begin{aligned}
& V_{\sigma\left(t_{j-1}\right)}^{1-\theta}(x(t)) \leq \phi^{j-1} e^{(1-\theta) q\left(t-t_{0}\right)} V_{\sigma\left(t_{0}\right)}^{1-\theta}\left(x\left(t_{0}\right)\right) \\
& +\frac{p}{q}\left(1-e^{(1-\theta) q\left(t-t_{j-1}\right)}\right) \\
& +\frac{p}{q} \phi\left(1-e^{(1-\theta) q\left(t_{j-1}-t_{j-2}\right)}\right) e^{(1-\theta) q\left(t-t_{j-1}\right)} \\
& +\frac{p}{q} \phi^{2}\left(1-e^{(1-\theta) q\left(t_{j-2}-t_{j-3}\right)}\right) e^{(1-\theta) q\left(t-t_{j-2}\right)} \\
& \ldots \\
& +\frac{p}{q} \phi^{j-2}\left(1-e^{(1-\theta) q\left(t_{2}-t_{1}\right)}\right) e^{(1-\theta) q\left(t-t_{2}\right)} \\
& +\frac{p}{q} \phi^{j-1}\left(1-e^{(1-\theta) q\left(t_{1}-t_{0}\right)}\right) e^{(1-\theta) q\left(t-t_{1}\right)} \\
& \quad \leq \phi^{j-1} e^{(1-\theta) q j \tau} V_{\sigma\left(t_{0}\right)}^{1-\theta}\left(x\left(t_{0}\right)\right) \\
& +\frac{p}{q} \frac{\phi\left(e^{(1-\theta) q \tau}-1\right)}{\phi e^{(1-\theta) q \tau}-1}\left(1-\phi^{j-1} e^{(1-\theta) q(j-1) \tau}\right) .
\end{aligned}
$$

Therefore, we obtain that

$$
0 \leq V_{\sigma\left(t_{j-1}\right)}^{1-\theta}(x(t)) \leq \Psi \phi^{j-1} e^{(1-\theta) q(j-1) \tau}+\Upsilon .
$$

It follows that

$$
0 \leq \Lambda(x(t)) \leq \frac{1}{\alpha^{1 / \mu}}\left(\Psi \phi^{j-1} e^{(1-\theta) q(j-1) \tau}+\Upsilon\right)^{1 /(1-\theta) \mu} .
$$


We can see that $\Psi<0$, which implies $\Lambda(x(t))=0$ for $j>1+$ $\left[\ln (-\Upsilon / \Psi) / \ln \left(\phi e^{(1-\theta) q \tau}\right)\right]$. Therefore, we conclude that $x(t)=$ 0 for $t>t_{\Pi}$.

\subsection{Stability with (9)}

Lemma 13. Let $t^{\prime \prime}>t^{\prime} \geq t_{0}$. If function $y(t): \mathbb{R} \longrightarrow \mathbb{R}$ satisfies

$$
\dot{y}(t)=p y^{\theta}(t)-q y(t)
$$

for $p>0, q>0$, and $0<\theta<1$, then one gets that

$$
\begin{aligned}
y^{1-\theta}\left(t^{\prime \prime}\right)= & e^{-(1-\theta) q\left(t^{\prime \prime}-t^{\prime}\right)} y^{1-\theta}\left(t^{\prime}\right) \\
& +\frac{p}{q}\left(1-e^{-(1-\theta) q\left(t^{\prime \prime}-t^{\prime}\right)}\right) .
\end{aligned}
$$

Proof. Multiplying (25) by $e^{-q t}$, we get that

$$
e^{q t} \dot{y}(y)+q e^{q t} y(t)=p e^{q t} y^{\theta}(t)
$$

which implies

$$
\frac{d\left(e^{q t} y(t)\right)}{d t}=p e^{q t} y^{\theta}(t) .
$$

Let $z(t)=e^{q t} y(t)$. Then, we have that

$$
\frac{d z}{d t}=p e^{(1-\theta) q t} z^{\theta} .
$$

Integrating (29) on the time interval $\left(t^{\prime}, t^{\prime \prime}\right)$, we get that

$$
z^{1-\theta}\left(t^{\prime \prime}\right)=z^{1-\theta}\left(t^{\prime}\right)+\frac{p}{q}\left(e^{(1-\theta) q t^{\prime \prime}}-e^{(1-\theta) q t^{\prime}}\right)
$$

which implies

$$
\begin{aligned}
y^{1-\theta}\left(t^{\prime \prime}\right)= & e^{-(1-\theta) q\left(t^{\prime \prime}-t^{\prime}\right)} y^{1-\theta}\left(t^{\prime}\right) \\
& +\frac{p}{q}\left(1-e^{-(1-\theta) q\left(t^{\prime \prime}-t^{\prime}\right)}\right) .
\end{aligned}
$$

Theorem 14. Consider switched system (1). Assume that Assumptions 1, 5, and 9 hold. Let $\Upsilon=(p / q)\left(e^{-(1-\theta) q \tau}-1\right) /$ $\left(\phi e^{-(1-\theta) q \tau}-1\right)$ and $\Psi=V_{\sigma\left(t_{0}\right)}^{1-\theta}\left(x\left(t_{0}\right)\right)-\Upsilon \phi$. Then, one can have the following:

(1) If $\phi e^{-(1-\theta) q \tau}-1<0$, then the states $x(t)$ of (1) will converge to set $\Omega$ globally as $t \longrightarrow \infty$, where $\Omega=\{x: \Lambda(x) \leq$ $\left.\left(1+\phi-\phi e^{-(1-\theta) q \tau}\right)(p / q)\left(e^{-(1-\theta) q \tau}-1\right) /\left(\phi e^{-(1-\theta) q \tau}-1\right)\right\}$.

(2) If $\phi e^{-(1-\theta) q \tau}-1>0$, then the origin of (1) is globally finite-time stable and the setting time is $t_{\Pi}$, where $\Pi=$ $\left[\ln \left(-\Upsilon\left(1+\phi-\phi e^{-(1-\theta) q \tau}\right) / \Psi\right) / \ln \left(e^{-(1-\theta) q \tau}\right)\right]$.

Proof. Using the homogeneous function $V_{i}(x), i \in \mathscr{P}$, we construct the multiple Lyapunov function $V_{\sigma(t)}(x)$ corresponding to switching law $\sigma(t)$. We can see that $V_{\sigma(t)}(x)$ is piecewise smooth.

For any $t \geq t_{0}$, there exists time interval $\left[t_{j-1}, t_{j}\right), j \geq 1$, such that $t_{j-1} \leq t<t_{j}$. Integrating differential inequalities (9) successively on the intervals $\left[t_{0}, t_{1}\right),\left[t_{1}, t_{2}\right), \ldots,\left[t_{j-1}, t\right]$, we can get

$$
\begin{aligned}
& V_{\sigma\left(t_{j-1}\right)}^{1-\theta}(x(t)) \leq e^{-(1-\theta) q\left(t-t_{j-1}\right)} V_{\sigma\left(t_{j-1}\right)}^{1-\theta}\left(x\left(t_{j-1}\right)\right) \\
& \quad+\frac{p}{q}\left(1-e^{-(1-\theta) q\left(t-t_{j-1}\right)}\right) \\
& V_{\sigma\left(t_{j-2}\right)}^{1-\theta}\left(x\left(t_{j-1}\right)\right) \leq e^{-(1-\theta) q\left(t_{j-1}-t_{j-2}\right)} V_{\sigma\left(t_{j-2}\right)}^{1-\theta}\left(x\left(t_{j-2}\right)\right) \\
& \quad+\frac{p}{q}\left(1-e^{-(1-\theta) q\left(t_{j-1}-t_{j-2}\right)}\right) \\
& \quad \ldots \\
& V_{\sigma\left(t_{0}\right)}^{1-\theta}\left(x\left(t_{1}\right)\right) \leq e^{-(1-\theta) q\left(t_{1}-t_{0}\right)} V_{\sigma\left(t_{0}\right)}^{1-\theta}\left(x\left(t_{0}\right)\right) \\
& \quad+\frac{p}{q}\left(1-e^{-(1-\theta) q\left(t_{1}-t_{0}\right)}\right) .
\end{aligned}
$$

Taking into account condition (5), we obtain

$$
V_{\sigma\left(t_{i}\right)}^{1-\alpha}\left(x\left(t_{i}\right)\right) \leq \phi V_{\sigma\left(t_{i-1}\right)}^{1-\alpha}\left(x\left(t_{i}\right)\right)
$$

for any $i=1, \ldots, j-1$. Substituting (33) into (32) yields

$$
\begin{aligned}
& V_{\sigma\left(t_{j-1}\right)}^{1-\theta}(x(t)) \leq \phi e^{-(1-\theta) q\left(t-t_{j-1}\right)} V_{\sigma\left(t_{j-2}\right)}^{1-\theta}\left(x\left(t_{j-1}\right)\right) \\
& \quad+\frac{p}{q}\left(1-e^{-(1-\theta) q\left(t-t_{j-1}\right)}\right) \\
& V_{\sigma\left(t_{j-2}\right)}^{1-\theta}\left(x\left(t_{j-1}\right)\right) \\
& \quad \leq \phi e^{-(1-\theta) q\left(t_{j-1}-t_{j-2}\right)} V_{\sigma\left(t_{j-3}\right)}^{1-\theta}\left(x\left(t_{j-2}\right)\right) \\
& \quad+\frac{p}{q}\left(1-e^{-(1-\theta) q\left(t_{j-1}-t_{j-2}\right)}\right) \\
& \ldots \\
& V_{\sigma\left(t_{0}\right)}^{1-\theta}\left(x\left(t_{1}\right)\right) \leq e^{-(1-\theta) q\left(t_{1}-t_{0}\right)} V_{\sigma\left(t_{0}\right)}^{1-\theta}\left(x\left(t_{0}\right)\right) \\
& \quad+\frac{p}{q}\left(1-e^{-(1-\theta) q\left(t_{1}-t_{0}\right)}\right) .
\end{aligned}
$$

It follows that

$$
\begin{aligned}
& V_{\sigma\left(t_{j-1}\right)}^{1-\theta}(x(t)) \leq \phi^{j-1} e^{-(1-\theta) q\left(t-t_{0}\right)} V_{\sigma\left(t_{0}\right)}^{1-\theta}\left(x\left(t_{0}\right)\right)+\frac{p}{q}(1 \\
& \left.-e^{-(1-\theta) q\left(t-t_{j-1}\right)}\right)+\frac{p}{q} \phi\left(1-e^{-(1-\theta) q\left(t_{j-1}-t_{j-2}\right)}\right) \\
& \quad \cdot e^{-(1-\theta) q\left(t-t_{j-1}\right)}+\frac{p}{q} \phi^{2}\left(1-e^{-(1-\theta) q\left(t_{j-2}-t_{j-3}\right)}\right) \\
& \quad \cdot e^{-(1-\theta) q\left(t-t_{j-2}\right)} \\
& \ldots \\
& +\frac{p}{q} \phi^{j-2}\left(1-e^{-(1-\theta) q\left(t_{2}-t_{1}\right)}\right) e^{-(1-\theta) q\left(t-t_{2}\right)}+\frac{p}{q} \phi^{j-1}(1 \\
& \left.\quad-e^{-(1-\theta) q\left(t_{1}-t_{0}\right)}\right) e^{-(1-\theta) q\left(t-t_{1}\right)}
\end{aligned}
$$




$$
\begin{aligned}
& \leq \phi^{j-1} e^{-(1-\theta) q(j-1) \tau} V_{\sigma\left(t_{0}\right)}^{1-\theta}\left(x\left(t_{0}\right)\right)+\frac{p}{q} \\
& \cdot \frac{e^{-(1-\theta) q \tau}-1}{\phi e^{-(1-\theta) q \tau}-1}\left(1+\phi-\phi e^{-(1-\theta) q \tau}\right. \\
& \left.-\phi^{j} e^{-(1-\theta) q(j-1) \tau}\right) .
\end{aligned}
$$

Therefore, we obtain that

$$
\begin{aligned}
0 & \leq V_{\sigma\left(t_{j-1}\right)}^{1-\theta}(x(t)) \\
& \leq \Psi \phi^{j-1} e^{-(1-\theta) q(j-1) \tau}+\Upsilon\left(1+\phi-\phi e^{-(1-\theta) q \tau}\right) .
\end{aligned}
$$

It follows that

$$
\begin{aligned}
0 \leq & \Lambda(x(t)) \leq \frac{1}{\alpha^{1 / \mu}}\left(\Psi \phi^{j-1} e^{-(1-\theta) q(j-1) \tau}\right. \\
& \left.+\Upsilon\left(1+\phi-\phi e^{-(1-\theta) q \tau}\right)\right)^{1 /(1-\theta) \mu} .
\end{aligned}
$$

Then, we consider the following two cases.

Case $1\left(\phi e^{-(1-\theta) q \tau}-1<0\right)$. We can see that $x(t)$ converges to $\Omega=\left\{x: \Lambda(x) \leq \Upsilon\left(1+\phi-\phi e^{-(1-\theta) q \tau}\right)\right\}$ as $t \longrightarrow \infty$.

Case $2\left(\phi e^{-(1-\theta) q \tau}-1>0\right)$. We can see that system (1) is globally finite-time stable, and the setting time is $t_{\Pi}$.

\subsection{Stability with (10)}

Lemma 15. Let $t^{\prime \prime}>t^{\prime} \geq t_{0}$. If function $y(t): \mathbb{R} \rightarrow \mathbb{R}$ satisfies

$$
\dot{y}(t)=-p y^{\theta}(t)-q y(t)
$$

for $p>0, q>0$, and $0<\theta<1$, then one gets that

$$
\begin{aligned}
y^{1-\theta}\left(t^{\prime \prime}\right)= & e^{-(1-\theta) q\left(t^{\prime \prime}-t^{\prime}\right)} y^{1-\theta}\left(t^{\prime}\right) \\
& -\frac{p}{q}\left(1-e^{-(1-\theta) q\left(t^{\prime \prime}-t^{\prime}\right)}\right) .
\end{aligned}
$$

Proof. Multiplying (25) by $e^{q t}$, we get that

$$
e^{q t} \dot{y}(y)+q e^{q t} y(t)=-p e^{q t} y^{\theta}(t)
$$

which implies

$$
\frac{d\left(e^{q t} y(t)\right)}{d t}=-p e^{q t} y^{\theta}(t) .
$$

Let $z(t)=e^{q t} y(t)$. Then, we have that

$$
\frac{d z}{d t}=-p e^{(1-\theta) q t} z^{\theta}
$$

Integrating (42) on the time interval $\left(t^{\prime}, t^{\prime \prime}\right)$, we get that

$$
z^{1-\theta}\left(t^{\prime \prime}\right)=z^{1-\theta}\left(t^{\prime}\right)-\frac{p}{q}\left(e^{(1-\theta) q t^{\prime \prime}}-e^{(1-\theta) q t^{\prime}}\right)
$$

which implies

$$
\begin{aligned}
y^{1-\theta}\left(t^{\prime \prime}\right)= & e^{-(1-\theta) q\left(t^{\prime \prime}-t^{\prime}\right)} y^{1-\theta}\left(t^{\prime}\right) \\
& -\frac{p}{q}\left(1-e^{-(1-\theta) q\left(t^{\prime \prime}-t^{\prime}\right)}\right) .
\end{aligned}
$$

Theorem 16. Consider switched system (1). Assume that Assumptions 1, 5, and 10 hold. Let $\Upsilon=(p)$ $q)\left(\phi e^{-(1-\theta) q \tau}\left(e^{-(1-\theta) q \tau}-1\right) /\left(\phi e^{-(1-\theta) q \tau}-1\right)\right)$ and $\Psi=$ $V_{\sigma\left(t_{0}\right)}^{1-\theta}\left(x\left(t_{0}\right)\right)+\Upsilon$. Then, one has the following:

(1) If $\phi e^{-(1-\theta) q \tau}-1<0$, then the origin of (1) is globally finite-time stable for any initial values.

(2) If $\phi e^{-(1-\theta) q \tau}-1>0$, then the origin of (1) is locally finite-time stable for initial value $x\left(t_{0}\right)$ satisfying $V_{\sigma\left(t_{0}\right)}^{1-\theta}\left(x\left(t_{0}\right)\right) \leq$ $-(p / q)\left(\phi e^{-(1-\theta) q \tau}\left(e^{-(1-\theta) q \tau}-1\right) /\left(\phi e^{-(1-\theta) q \tau}-1\right)\right)$.

Moreover, the setting times for cases (1) and (2) are both $t_{\Pi}$, where $\Pi=\left[\ln (\Upsilon / \Psi) / \ln \left(\phi / e^{(1-\theta) q \tau}\right)\right]$.

Proof. Using the homogeneous function $V_{i}(x), i \in \mathscr{P}$, we construct the multiple Lyapunov function $V_{\sigma(t)}(x)$ corresponding to switching law $\sigma(t)$. We can see that $V_{\sigma(t)}(x)$ is piecewise smooth.

For any $t \geq t_{0}$, there exists time interval $\left[t_{j-1}, t_{j}\right), j \geq 1$, such that $t_{j-1} \leq t<t_{j}$. Integrating differential inequalities (10) successively on the intervals $\left[t_{0}, t_{1}\right),\left[t_{1}, t_{2}\right), \ldots,\left[t_{j-1}, t\right]$, we can get

$$
\begin{aligned}
& V_{\sigma\left(t_{j-1}\right)}^{1-\theta}(x(t)) \leq e^{-(1-\theta) q\left(t-t_{j-1}\right)} V_{\sigma\left(t_{j-1}\right)}^{1-\theta}\left(x\left(t_{j-1}\right)\right) \\
& \quad-\frac{p}{q}\left(1-e^{-(1-\theta) q\left(t-t_{j-1}\right)}\right) \\
& V_{\sigma\left(t_{j-2}\right)}^{1-\theta}\left(x\left(t_{j-1}\right)\right) \leq e^{-(1-\theta) q\left(t_{j-1}-t_{j-2}\right)} V_{\sigma\left(t_{j-2}\right)}^{1-\theta}\left(x\left(t_{j-2}\right)\right) \\
& \quad-\frac{p}{q}\left(1-e^{-(1-\theta) q\left(t_{j-1}-t_{j-2}\right)}\right) \\
& \quad . \quad \\
& V_{\sigma\left(t_{0}\right)}^{1-\theta}\left(x\left(t_{1}\right)\right) \leq e^{-(1-\theta) q\left(t_{1}-t_{0}\right)} V_{\sigma\left(t_{0}\right)}^{1-\theta}\left(x\left(t_{0}\right)\right) \\
& \quad-\frac{p}{q}\left(1-e^{-(1-\theta) q\left(t_{1}-t_{0}\right)}\right) .
\end{aligned}
$$

Taking into account condition (5), we obtain

$$
V_{\sigma\left(t_{i}\right)}^{1-\alpha}\left(x\left(t_{i}\right)\right) \leq \phi V_{\sigma\left(t_{i-1}\right)}^{1-\alpha}\left(x\left(t_{i}\right)\right)
$$

for any $i=1, \ldots, j-1$. Substituting (46) into (45) yields

$$
\begin{aligned}
& V_{\sigma\left(t_{j-1}\right)}^{1-\theta}(x(t)) \leq \phi e^{-(1-\theta) q\left(t-t_{j-1}\right)} V_{\sigma\left(t_{j-2}\right)}^{1-\theta}\left(x\left(t_{j-1}\right)\right) \\
& \quad-\frac{p}{q}\left(1-e^{-(1-\theta) q\left(t-t_{j-1}\right)}\right) \\
& V_{\sigma\left(t_{j-2}\right)}^{1-\theta}\left(x\left(t_{j-1}\right)\right) \\
& \quad \leq \phi e^{-(1-\theta) q\left(t_{j-1}-t_{j-2}\right)} V_{\sigma\left(t_{j-3}\right)}^{1-\theta}\left(x\left(t_{j-2}\right)\right)
\end{aligned}
$$




$$
\begin{aligned}
& \quad-\frac{p}{q}\left(1-e^{-(1-\theta) q\left(t_{j-1}-t_{j-2}\right)}\right) \\
& \ldots \\
& V_{\sigma\left(t_{0}\right)}^{1-\theta}\left(x\left(t_{1}\right)\right) \leq e^{-(1-\theta) q\left(t_{1}-t_{0}\right)} V_{\sigma\left(t_{0}\right)}^{1-\theta}\left(x\left(t_{0}\right)\right) \\
& \quad-\frac{p}{q}\left(1-e^{-(1-\theta) q\left(t_{1}-t_{0}\right)}\right) .
\end{aligned}
$$

It follows that

$$
\begin{aligned}
& V_{\sigma\left(t_{j-1}\right)}^{1-\theta}(x(t)) \leq \phi^{j-1} e^{-(1-\theta) q\left(t-t_{0}\right)} V_{\sigma\left(t_{0}\right)}^{1-\theta}\left(x\left(t_{0}\right)\right) \\
& -\frac{p}{q}\left(1-e^{-(1-\theta) q\left(t-t_{j-1}\right)}\right)-\frac{p}{q} \\
& \cdot \phi\left(1-e^{-(1-\theta) q\left(t_{j-1}-t_{j-2}\right)}\right) e^{-(1-\theta) q\left(t-t_{j-1}\right)}-\frac{p}{q} \\
& \cdot \phi^{2}\left(1-e^{-(1-\theta) q\left(t_{j-2}-t_{j-3}\right)}\right) e^{-(1-\theta) q\left(t-t_{j-2}\right)} \\
& \cdots \cdot \frac{p}{q} \phi^{j-2}\left(1-e^{-(1-\theta) q\left(t_{2}-t_{1}\right)}\right) e^{-(1-\theta) q\left(t-t_{2}\right)}-\frac{p}{q} \\
& \cdot \phi^{j-1}\left(1-e^{-(1-\theta) q\left(t_{1}-t_{0}\right)}\right) e^{-(1-\theta) q\left(t-t_{1}\right)} \\
& \leq \phi^{j-1} e^{-(1-\theta) q(j-1) \tau} V_{\sigma\left(t_{0}\right)}^{1-\theta}\left(x\left(t_{0}\right)\right)-\frac{p}{q} \\
& \quad \frac{\phi e^{-(1-\theta) q \tau}\left(e^{-(1-\theta) q \tau}-1\right)}{\phi e^{-(1-\theta) q \tau}-1}\left(1-\phi^{j-1} e^{-(1-\theta) q(j-1) \tau}\right) .
\end{aligned}
$$

Therefore, we obtain that

$$
0 \leq V_{\sigma\left(t_{j-1}\right)}^{1-\theta}(x(t)) \leq \Psi \phi^{j-1} e^{-(1-\theta) q(j-1) \tau}-\Upsilon .
$$

It follows that

$$
\begin{aligned}
0 & \leq \Lambda(x(t)) \\
& \leq \frac{1}{\alpha^{1 / \mu}}\left(\Psi \phi^{j-1} e^{-(1-\theta) q(j-1) \tau}-\Upsilon\right)^{1 /(1-\theta) \mu} .
\end{aligned}
$$

Then, we consider the following two cases.

Case $1\left(\phi e^{-(1-\theta) q \tau}-1<0\right)$. We can see that the switched system (1) is globally finite-time stable with any initial value and the setting time is $t_{\Pi}$.

Case $2\left(\phi e^{-(1-\theta) q \tau}-1>0\right)$. We can see that if $\Psi<0$, then switched system (1) is locally finite-time stable and the setting time also is $t_{\Pi}$.

\section{Numerical Example}

Consider the following switched system:

$$
f_{1}(x)=\left(\begin{array}{c}
-x_{1}-x_{1}^{7 / 9}-x_{2}^{3} \\
x_{1}^{1 / 3}-\frac{1}{3^{5 / 6}} x_{2}^{1 / 3}-\frac{1}{3} x_{2}
\end{array}\right)
$$
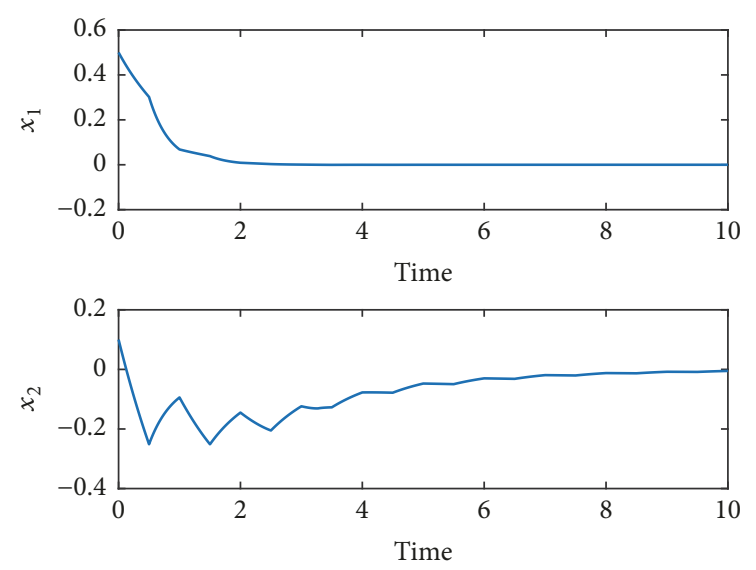

FIGURE 1: State trajectories of $x$.

$$
f_{2}(x)=\left(\begin{array}{c}
-3 x_{2}^{3} x_{1}^{2 / 3}-6 x_{1}^{5 / 7}-3 x_{1} \\
x_{1}-2 x_{2}^{1 / 7}-x_{2}
\end{array}\right)
$$

The sequence of switching times is given by $t_{0}=0, t_{k}=$ $t_{k-1}+0.5$. Choose $V_{1}(x)=3 x_{1}^{4 / 3}+x_{2}^{4}$ and $V_{2}(x)=x_{1}^{4 / 3}+x_{2}^{4}$. We get that $V_{1}(x)$ and $V_{2}(x)$ are both homogeneous of degree $\mu=4$ with respect to $r=(3,1)^{T}$. The derivatives of the homogeneous functions are $\nabla V_{1}(x) f_{1}(x) \leq-(4 / 3) V_{1}(x)-$ $(4 / 3) V_{1}^{5 / 6}(x)$ and $\nabla V_{2}(x) f_{2}(x) \leq-4 V_{2}(x)-8 V_{2}^{5 / 6}(x)$. Then, we obtain that $p=q=4 / 3, \theta=5 / 6$, and $\phi=3$. According to Theorem 16, we get that switched system (51) is finite-time stable. Figure 1 shows the state responses, which demonstrate the exactness of the results.

\section{Conclusion}

In this paper, the finite-time stability problem of switched homogeneous systems has been studied. Three substantially different situations with hybrid homogeneous degrees have been introduced. Sufficient conditions and estimations of the setting time have been given under both situations. A numerical example has been presented to show the effectiveness of the results.

\section{Data Availability}

The data used to support the findings of this study are included within the article.

\section{Conflicts of Interest}

The author declares that there are no conflicts of interest regarding the publication of this paper.

\section{Acknowledgments}

This work was supported by the National Natural Science Foundation of China (61603050) and the Fundamental Research Funds for the Central Universities (2017RC25). 


\section{References}

[1] R. Cardim, M. C. M. Teixeira, E. Assunção, and M. R. Covacic, "Variable-structure control design of switched systems with an application to a DC-DC power converter," IEEE Transactions on Industrial Electronics, vol. 56, no. 9, pp. 3505-3513, 2009.

[2] R. Olfati-Saber and R. M. Murray, "Consensus problems in networks of agents with switching topology and time-delays," IEEE Transactions on Automatic Control, vol. 49, no. 9, pp. 15201533,2004

[3] Y. Jia, "Robust control with decoupling performance for steering and traction of 4WS vehicles under velocity-varying motion," IEEE Transactions on Control Systems Technology, vol. 8, no. 3, pp. 554-569, 2000.

[4] Y. Jia, "Alternative proofs for improved LMI representations for the analysis and the design of continuous-time systems with polytopic type uncertainty: A predictive approach," Institute of Electrical and Electronics Engineers Transactions on Automatic Control, vol. 48, no. 8, pp. 1413-1416, 2003.

[5] D. Liberzon and A. S. Morse, "Basic problems in stability and design of switched systems," IEEE Control Systems Magazine, vol. 19, no. 5, pp. 59-70, 1999.

[6] D. Liberzon, Switching in Cystems and Control, Birkhäuser, Boston, Mass, USA, 2003.

[7] Z. Sun and S. S. Ge, Switched linear systems: Control and Design, Spronger, London, UK, 2005.

[8] K. S. Narendra and J. A. Balakrishnan, "A common Lyapunov function for stable LTI systems with commuting A-matrices," IEEE Transactions on Automatic Control, vol. 39, no. 12, pp. 2469-2471, 1994.

[9] L. Vu and D. Liberzon, "Common Lyapunov functions for families of commuting nonlinear systems," Systems \& Control Letters, vol. 54, no. 5, pp. 405-416, 2005.

[10] M. S. Branicky, "Multiple Lyapunov functions and other analysis tools for switched and hybrid systems," IEEE Transactions on Automatic Control, vol. 43, no. 4, pp. 475-482, 1998.

[11] A. Bacciotti and L. Mazzi, "An invariance principle for nonlinear switched systems," Systems \& Control Letters, vol. 54, no. 11, pp. 1109-1119, 2005.

[12] B. Zhang and Y. Jia, "On weak-invariance principles for nonlinear switched systems," Institute of Electrical and Electronics Engineers Transactions on Automatic Control, vol. 59, no. 6, pp. 1600-1605, 2014

[13] A. Levant, D. Efimov, A. Polyakov, and W. Perruquetti, "Stability and robustness of homogeneous differential inclusions," in Proceedings of the 55nd IEEE Conference on Decision and Control, pp. 7288-7293, Las Vegas, NV, USA, 2016.

[14] V. Andrieu, L. Praly, and A. Astolfi, "Homogeneous approximation, recursive observer design, and output feedback," SIAM Journal on Control and Optimization, vol. 47, no. 4, pp. 18141850, 2008.

[15] L. Zhang, S. Liu, and H. Lan, "On stability of switched homogeneous nonlinear systems," Journal of Mathematical Analysis and Applications, vol. 334, no. 1, pp. 414-430, 2007.

[16] A. Y. Aleksandrov, A. A. Kosov, and A. V. Platonov, "On the asymptotic stability of switched homogeneous systems," Systems \& Control Letters, vol. 61, no. 1, pp. 127-133, 2012.

[17] R. Kuiava, B. K. Matos, and G. . Razente, "Finite-time stability of a class of continuous-time non-homogeneous switched systems," Nonlinear Analysis: Hybrid Systems, vol. 26, pp. 101114, 2017.
[18] D. Holcman and M. Margaliot, "Stability analysis of secondorder switched homogeneous systems," SIAM Journal on Control and Optimization, vol. 41, no. 5, pp. 1609-1625, 2002.

[19] Y. Orlov, "Finite time stability of homogeneous switched systems," in Proceedings of the 42nd IEEE International Conference on Decision and Control, pp. 4271-4276, Maui, HI, USA, 2003.

[20] Y. Shen and X. Xia, "Semi-global finite-time observers for nonlinear systems," Automatica, vol. 44, no. 12, pp. 3152-3156, 2008.

[21] Y. Shen, Y. Huang, and J. Gu, "Global finite-time observers for lipschitz nonlinear systems," IEEE Transactions on Automatic Control, vol. 56, no. 2, pp. 418-424, 2011.

[22] E. Bernuau, A. Polyakov, D. Efimov, and W. Perruquetti, "Robustness of finite-time stability property for sliding modes," IFAC Proceedings Volumes, vol. 46, no. 2, pp. 391-396, 2013.

[23] E. Zavala and J. A. Moreno, "Improved convergence rate of discontinuous finite-time controllers," IFAC Proceedings Volumes, vol. 47, no. 3, pp. 8636-8641, 2014. 


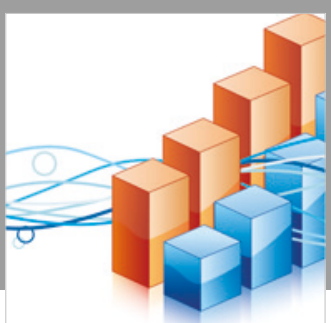

Advances in

Operations Research

\section{-n-m}
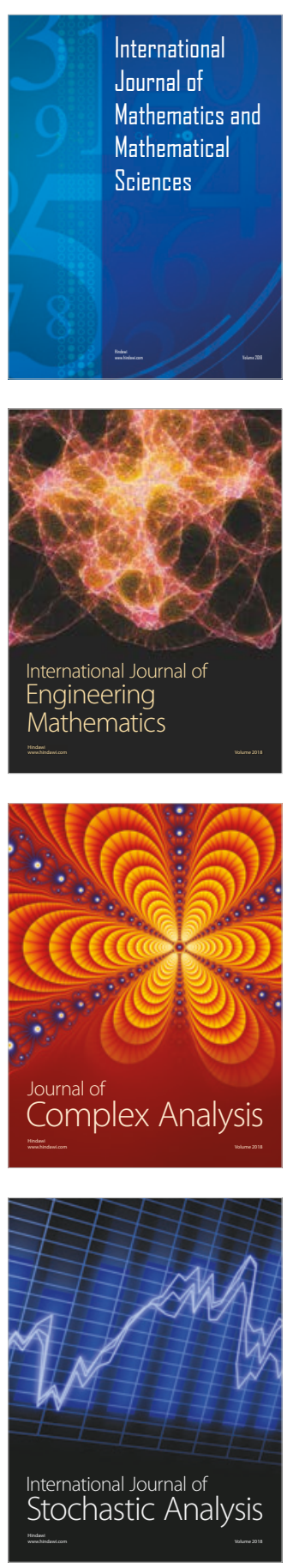
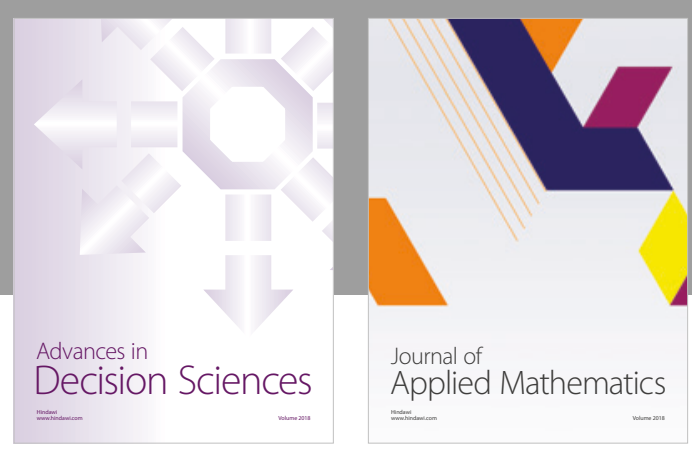

Journal of

Applied Mathematics
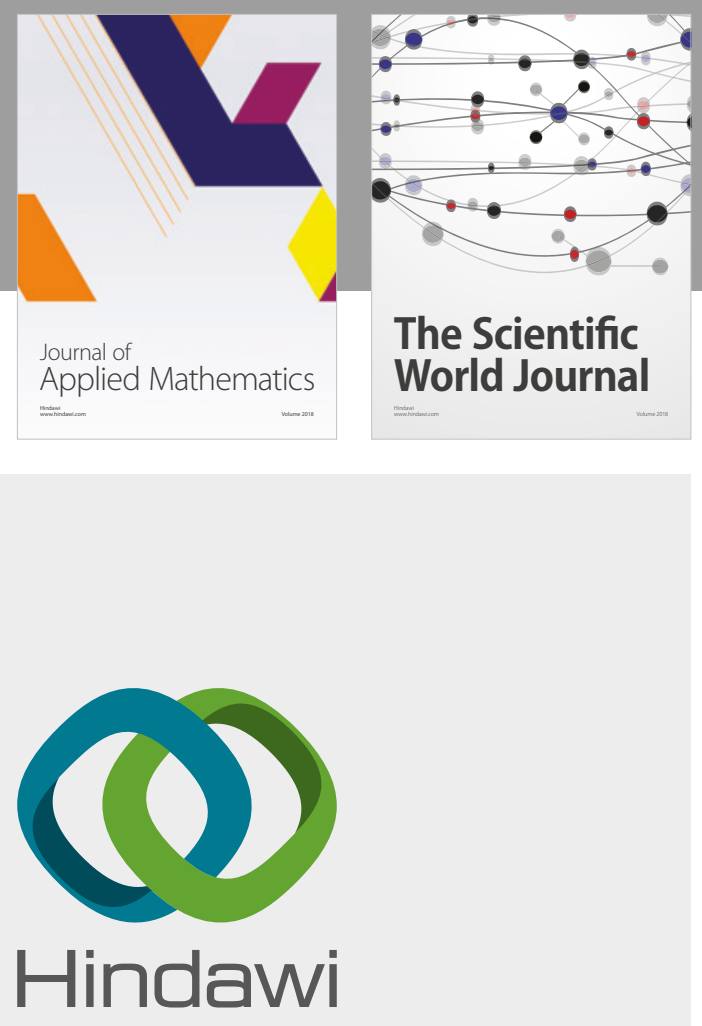

Submit your manuscripts at

www.hindawi.com

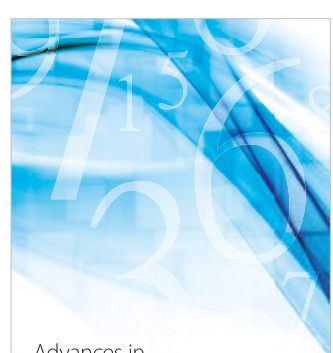

Advances in
Numerical Analysis
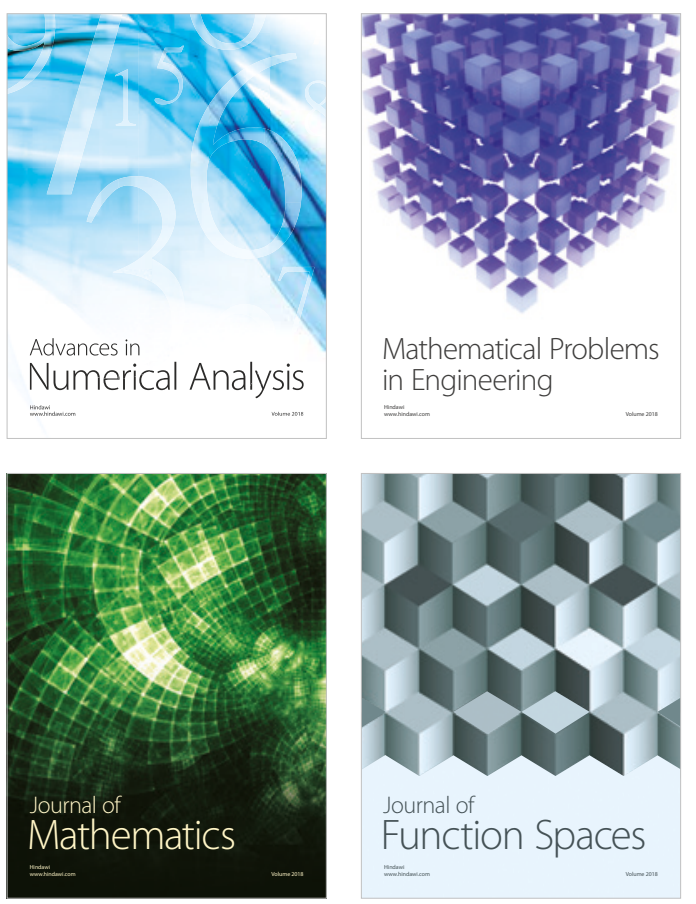

Mathematical Problems in Engineering

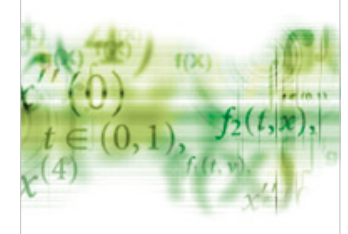

International Journal of

Differential Equations

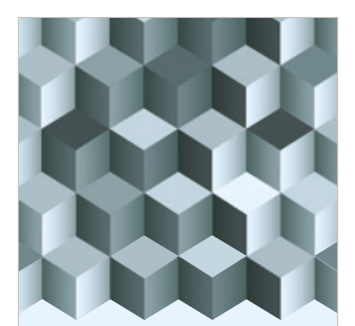

Journal of

Function Spaces

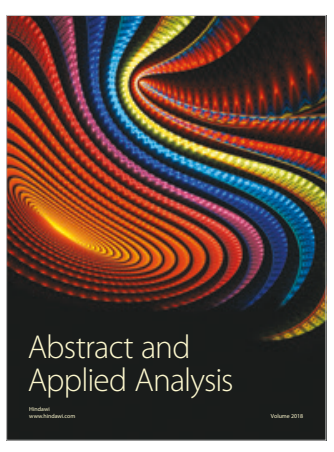

The Scientific

World Journal

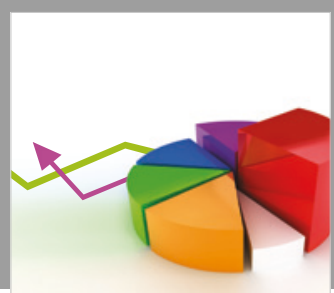

Journal of

Probability and Statistics
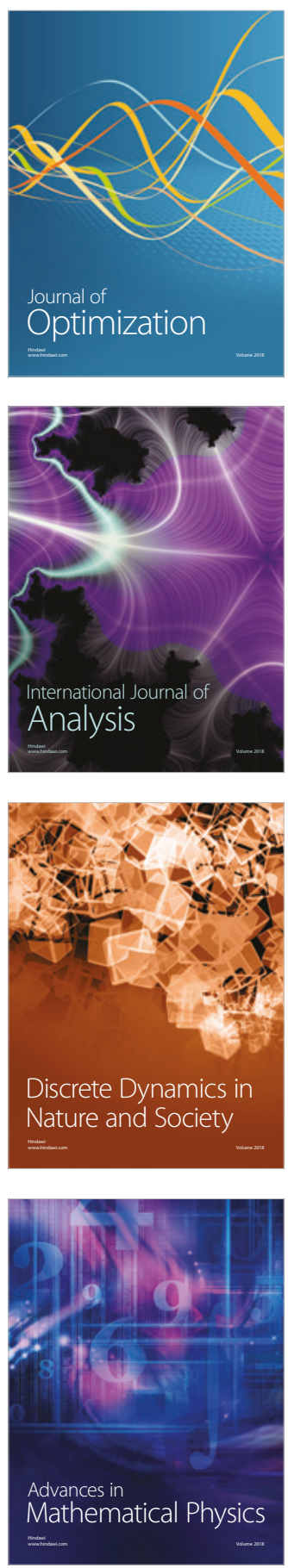